\title{
Effects of Procainamide and Disopyramide on Long Ghain Acyl Carnitine and Long Ghain Acyl CoA Concentrations in the Ischemic Heart
}

\author{
Akira Kobayashi, M.D., Hiroshi Watanabe, M.D., \\ and Noboru Yamazaki, M.D.
}

\begin{abstract}
SUMMARY
Though the efficacies of procainamide and disopyramide in treating arrhythmias are well established, their precise mechanisms of antiarrhythmic action remain unclear. Arrhythmias which occur during acute myocardial ischemia can be explained partly on a metabolic basis. The accumulation of intermediates subsequent to impaired $\beta$-oxidation of free fatty acids has been suggested as a cause of serious arrhythmias. The purpose of this study was to investigate changes in free carnitine, long chain acyl carnitine and long chain acyl CoA concentrations in the ischemic canine heart following the administration of procainamide and disopyramide. The coronary artery was occluded for $40 \mathrm{~min}$ and myocardial samples were prepared from both nonischemic and ischemic areas. Procainamide and disopyramide prevented the accumulation of long chain acyl carnitine and long chain acyl $\mathrm{CoA}$ in the ischemic myocardium. The results showed that procainamide and disopyramide had beneficial effects on fatty acid metabolism. It was suggested that one of the antiarrhythmic mechanisms of these drugs might be the prevention of the accumulation of fatty acyl derivatives in the ischemic myocardium.
\end{abstract}

\section{Additional Indexing Words:}

Free fatty acids Carnitine Ventricular arrhythmias Antiarrhythmic drugs Antiarrhythmic mechanism Cyclic AMP

$\mathrm{V}$ ENTRIGULAR arrhythmias have been reported to account for many sudden deaths in patients with myocardial infarction. The efficacy of antiarrhythmic drugs in treating ventricular arrhythmias is well established. However, their precise mechanisms of antiarrhythmic action remain unclear. Recently, increasing emphasis has been placed on biochemical factors in the genesis of arrhythmias, and some arrhythmias occurring during acute myocardial ischemia can be explained on a metabolic basis. Much attention has

From the Third Department of Internal Medicine, Hamamatsu University School of Medicine, Hamamatsu, Shizuoka, Japan.

Address for reprints: Akira Kobayashi, M.D., Third Department of Internal Medicine, Hamamatsu University School of Medicine, 3600 Handa-cho, Hamamatsu, Shizuoka 431-31, Japan.

Received for publication May 27, 1987.

Accepted May 31, 1988. 
been focused on the arrhythmogenicity of free fatty acids (FFA) under conditions of ischemia from many clinical and experimental observations. An increased concentration of circulating FFA has been reported in patients with acute myocardial infarction, and elevated concentrations of FFA have been correlated with the appearance of ventricular arrhythmias. ${ }^{1)}$ In the ischemic heart, elevated FFA leads to the accumulation of fatty acyl derivatives, such as long chain acyl carnitine and long chain acyl $\mathrm{CoA}$ as a result of the inhibition of $\beta$-oxidation of FFA. ${ }^{2}$ Accumulation of FFA and its intermediates has been advocated as a cause of serious cardiac arrhythmias because of their direct toxic effects on the cell membrane, perhaps with structural changes in the ionic channels. ${ }^{3)}$ Sodium-2-[5-(4-chlorophenyl)-pentyl]-oxirane-2-carboxylate (POCA) is an inhibitor of carnitine acyltransferase $I$ and prevents the increase in long chain acyl carnitine in hypoxic cells. ${ }^{4}$ Pretreatment with POCA markedly attenuated the electrophysiologic derangements induced by hypoxia as assessed by intracellular transmembrane action potential recordings. ${ }^{4}$ Thus, it is likely that the inhibition of the accumulation of intermediates of FFA might have a beneficial effect on the prevention of arrhythmias associated with myocardial ischemia. Cyclic AMP accelerates lipolysis both in adipose tissue and in the heart. Propranolol decreases the infarct size and improves cardiac metabolism in acute ischemia, and one identified mechanism of its protective effects is the inhibition of lipolysis and the reduction of circulating FFA concentrations through alteration of the cyclic AMP system. ${ }^{5)}$ Quinidine, procainamide and disopyramide have been reported to reduce atrial cyclic AMP concentrations in guinea pigs and to inhibit hormone-stimulated lipolysis in isolated fat cells. ${ }^{61,7)}$ Although local anesthetics presumably act to stabilize the cell membrane and prevent arrhythmias, ${ }^{8)}$ they may also block the effect of cyclic AMP on lipid metabolism. However, there is no report available regarding the effects of antiarrhythmic drugs on the intermediates of FFA in the ischemic heart. Therefore, an investigation was undertaken to study the changes in the myocardial concentrations of long chain acyl carnitine and long chain acyl CoA following the administration of procainamide and disopyramide.

\section{Materials and Methods}

Twenty-four mongrel dogs of both sexes, weighing from 10 to $15 \mathrm{~kg}$, were used. All dogs were anesthetized with a bolus injection of sodium pentobarbital $(30 \mathrm{mg} / \mathrm{kg}$ i.v.). The trachea was intubated and respired with room air using a Harvard respirator. Thoracotomy was performed and the heart suspended in a pericardial cradle. The left anterior descending coro- 
nary artery was dissected free from surrounding tissue just distal to the origin of the first diagonal branch and a 1-0 silk thread was placed around the left anterior descending coronary artery. The coronary artery was occluded with an intracranial arterial clamp for $40 \mathrm{~min}$. Soon after the onset of coronary artery occlusion, the ischemic area was clearly apparent as a sharply delineated cyanotic area. Animals which did not exhibit epicardial cyanosis over the left ventricle were excluded from this study. Lead II of the standard electrocardiogram was recorded continuously during the experiment. Twentyfour dogs were divided into 3 groups as follows. The control group $(n=8)$ received isotonic saline solution. The procainamide-treated group $(n=8)$ was intravenously injected at a dose of $20 \mathrm{mg} / \mathrm{kg} 30 \mathrm{~min}$ prior to coronary occlusion followed by an infusion of $0.1 \mathrm{mg} / \mathrm{kg} / \mathrm{min}$ to the end of the experiment. The disopyramide-treated group $(n=8)$ was intravenously injected at a dose of $5 \mathrm{mg} / \mathrm{kg} 30 \mathrm{~min}$ prior to coronary occlusion followed by an infusion of 0.1 $\mathrm{mg} / \mathrm{kg} / \mathrm{min}$ to the end of the experiment. The doses of both agents were based on previous reports. ${ }^{9), 10}$ After 40 min of coronary artery occlusion, the beating hearts were immediately removed and transmural samples representing the ischemic area (supplied by the occluded left coronary artery) and nonischemic area (supplied by the circumflex coronary artery) were rapidly excised. Tissue samples were immediately frozen with a Wollenberger clamp which was cooled to the temperature of liquid nitrogen and then stored at $-80^{\circ} \mathrm{G}$. The animals which developed ventricular fibrillation were excluded from the analysis of metabolism because of different durations of ischemia. The concentration of free carnitine was measured by coupled enzymatic assays following the method of Marquis and Fritz. ${ }^{11}$ The concentration of long chain acyl CoA was assayed as free CoA after alkaline hydrolysis at $\mathrm{pH} 11.5$ to 12.0 in the presence of dithiothreitol and measured by the enzymatic cycling method of Veloso and Veech. ${ }^{12}$ ) We have reported the assay methods for frec carnitine, long chain acyl carnitine and long chain acyl CoA in another report. ${ }^{13)}$ In order to analyze their frequency and severity, ventricular arrhythmias were quantified by an arbitrary scoring system that was devised based on a modification of Lown and Wolf's ventricular arrhythmia grading system $^{14)}$ (Table I). Maximum scores were recorded in individual dogs every $5 \mathrm{~min}$ and added in each group. Values of the metabolites were expressed as mean $\pm \mathrm{SD}$ and statistical analysis was done using Student's t-test. Statistical analysis of ventricular arrhythmias was done by the Chi-square method and the Wilcoxon signed rank test. P values of less than 0.05 were considered significant. 
Table 1. Scoring System for Ventricular Arrhythmias

\begin{tabular}{l|l}
\hline Grade & \multicolumn{1}{c}{ Arrhythmias } \\
\hline 0 & No VPB \\
2 & Isolated uniform VPBs $<5 /$ min \\
3 & Isolated uniform VPBs $>5 / \mathrm{min}$ \\
4 & Multiform VPBs \\
5 & Couplets or salvos VPBs \\
6 & Ventricular tachycardia (VT*) \\
Ventricular fibrillation (VF) \\
VPB=ventricular premature beat.
\end{tabular}

\section{Results}

1. Effects on ventricular arrhythmias

In the control group, $1 \mathrm{dog}$ had ventricular fibrillation and 2 dogs had ventricular tachycardia during coronary artery occlusion. On the other hand, no dog showed ventricular fibrillation or ventricular tachycardia in the procainamide-treated and disopyramide-treated groups. Figure 1 shows the changes in the mean score of ventricular arrhythmias. The mcan scores at 30,35 and $40 \mathrm{~min}$ after coronary occlusion in the procainamide-treated and disopyramide-treated groups were significantly lower than those in the control group.

2. Myocardial concentrations of free carnitine

Figure 2 shows the myocardial concentrations of free carnitine in the ischemic and nonischemic areas in each group. In the control group, mean free carnitine concentrations in the ischemic and nonischemic areas were $632 \pm 223 \mathrm{nmol} / \mathrm{g}$ wet tissue and $1072 \pm 350 \mathrm{nmol} / \mathrm{g}$ wet tissue, respectively. In the procainamide-treated group, mean free carnitine concentrations in the ischemic and nonischemic areas were $671 \pm 129 \mathrm{nmol} / \mathrm{g}$ wet tissue and $1025 \pm$ $103 \mathrm{nmol} / \mathrm{g}$ wet tissue, respectively. In the disopyramide-treated group, mean free carnitine concentrations in the ischemic and nonischemic areas were $840 \pm$ $46 \mathrm{nmol} / \mathrm{g}$ wet tissue and $994 \pm 105 \mathrm{nmol} / \mathrm{g}$ wet tissue, respectively. The concentration of free carnitine in ischemic areas decreascd significantly compared with that in nonischemic areas in all groups. In ischemic areas, the concentration of free carnitine in the disopyramide-treated group was kept at a higher level than that in the control group $(p<0.05)$, but there was no significant difference between procainamide-treated and control groups.

3. Myocardial concentrations of long chain acyl carnitine

Figure 3 shows the myocardial concentrations of long chain acyl car- 


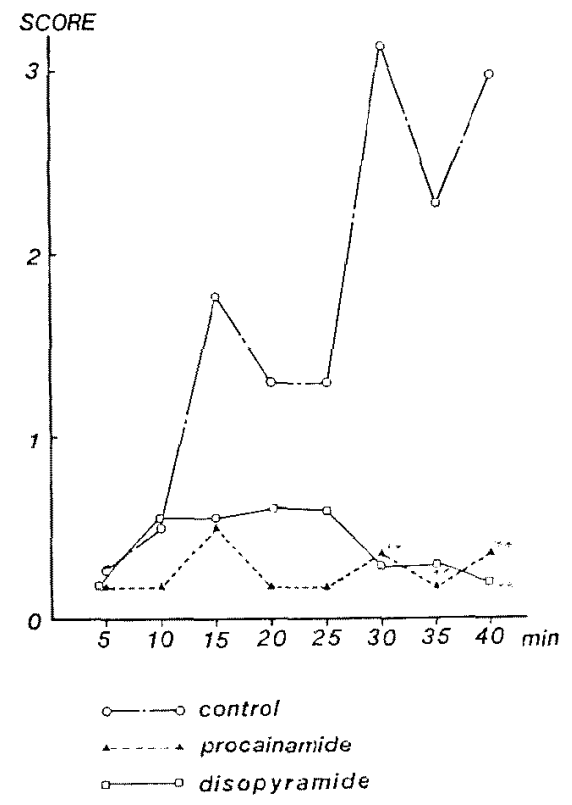

Fig. 1. Changes in the mean scores for ventricular arrhythmias during myocardial ischemia. Asterisks represent the significance of changes between the control group and the treated group. $* * \mathrm{p}<0.0 \mathrm{I}$.

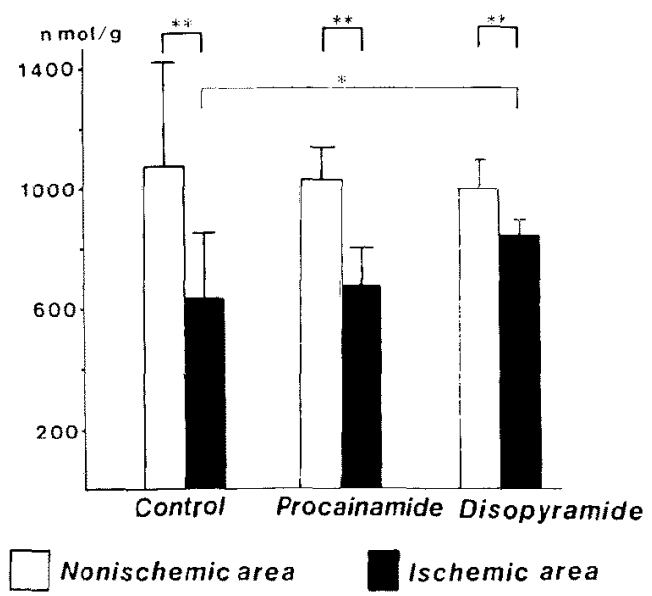

Fig. 2. Effects of procainamide and disopyramide on tissue levels of free carnitine. The significance of the changes is represented as follows: nonischemic area vs ischemic area (paired t-test), the control group vs treated group (nonpaired t-test). $* p<0.05, * * p<0.01$. 


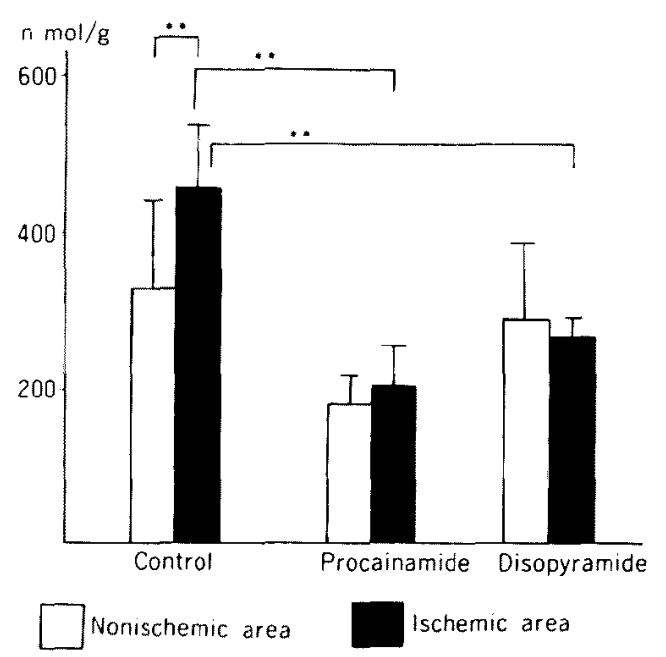

Fig. 3. Effects of procainamide and disopyramide on tissue levels of long chain acyl carnitine. The significance of the changes is represented as follows: nonischemic area vs ischemic area (paired t-test), the control group vs treated group (nonpaired t-test). ${ }^{* *} \mathrm{p}<0.01$.

nitine in the ischemic and nonischemic areas in each group. In the control group, mean long chain acyl carnitine concentrations in the ischemic and nonischemic areas were $458 \pm 80 \mathrm{nmol} / \mathrm{g}$ wet tissue and $331 \pm 102 \mathrm{nmol} / \mathrm{g}$ wet tissue, respectively. In the procainamide-treated group, mean long chain acyl carnitine concentrations in the ischemic and nonischemic areas were $203 \pm 51 \mathrm{nmol} / \mathrm{g}$ wet tissue and $186 \pm 23 \mathrm{nmol} / \mathrm{g}$ wet tissue, respectively. In the disopyramide-treated group, mean long chain acyl carnitine concentrations in the ischemic and nonischemic areas were $268 \pm 62 \mathrm{nmol} / \mathrm{g}$ wet tissue and $291 \pm 96 \mathrm{nmol} / \mathrm{g}$ wet tissue, respectively. The concentration of long chain acyl carnitine in the control group was significantly higher in the ischemic area than in the nonischemic area $(p<0.01)$. On the other hand, there was no significant difference between the ischemic and nonischemic areas in the procainamide-treated and disopyramide-treated groups. In ischemic areas, the concentrations of long chain acyl carnitine in the procainamide-treated and disopyramide-treated groups were significantly lower than those in the control group $(\mathrm{p}<0.01, \mathrm{p}<0.01)$. This shows that procainamide and disopyramide prevented the accumulation of long chain acyl carnitine in ischemic myocardium.

4. Myocardial concentrations of long chain acyl CoA

Figure 4 shows the myocardial concentrations of long chain acyl CoA in the ischemic and nonischemic areas in each group. In the control group, mean long chain acyl CoA concentrations in the ischemic and nonischemic 


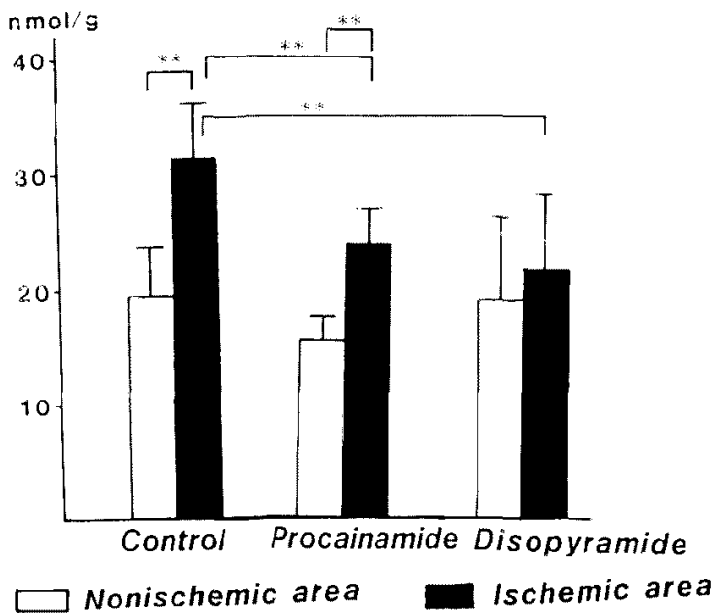

Fig. 4. Effects of procainamide and disopyramide on tissue levels of long chain acyl CoA. The significance of the changes is represented as follows: nonischemic area vs ischemic area (paired t-test), the control group vs treated group (nonpaired t-test). ** $\mathrm{p}<0.01$.

areas were $31.2 \pm 4.9 \mathrm{nmol} / \mathrm{g}$ wet tissue and $19.4 \pm 4.4 \mathrm{nmol} / \mathrm{g}$ wet tissue, respectively. In the procainamide-treated group, mean long chain acyl CoA concentrations in the ischemic and nonischemic areas were $23.7 \pm 2.7 \mathrm{nmol} / \mathrm{g}$ wet tissue and $15.7 \pm 2.1 \mathrm{nmol} / \mathrm{g}$ wet tissue, respectively. In the disopyramidetreated group, mean long chain acyl CoA concentrations in the ischemic and nonischemic areas were $21.9 \pm 7.4 \mathrm{nmol} / \mathrm{g}$ wet tissue and $19.5 \pm 7.0 \mathrm{nmol} / \mathrm{g}$ wet tissue, respectively. The concentrations of long chain acyl CoA in the control and procainamide-treated groups were significantly higher in the ischemic areas than in the nonischemic areas $(p<0.01, p<0.01)$. However, the concentration of long chain acyl $\mathrm{CoA}$ in the procainamide-treated group was significantly lower than that in the control in the ischemic area $(\mathrm{p}<0.01)$. On the other hand, there was no significant difference in the concentration of long chain acyl CoA between ischemic and nonischemic areas of the disopyramide-treated group. The concentration of long chain acyl CoA in the disopyramide-treated group was significantly lower than that in the control group in the ischemic area $(\mathrm{p}<0.01)$. This shows that disopyramide and procainamide prevented the accumulation of long chain acyl $\mathrm{CoA}$ in the ischemic myocardium.

\section{Discussion}

The efficacies of procainamide and disopyramide in treating ventricular 
arrhythmias are well established. Both agents are thought to be capable of suppressing automatic and reentrant arrhythmias, since they modify refractoriness and slow conduction, and suppress automaticity in Purkinje fibers and ventricular muscle cells. ${ }^{15}$ However, their precise mechanisms of antiarrhythmic action remain unclear. Arrhythmias which occur during acute myocardial hypoxia can be partly explained on a metabolic basis. Increased levels of circulating FFA have been reported in patients with acute myocardial infarction, ${ }^{11}$ and elevated levels of FFA have been correlated with the appearance of ventricular arrhythmias. ${ }^{2}$ ) An increased release of catecholamine is prominent in the ischemic heart and can lead to an increase in lipolysis and to an enhancement of the uptake of FFA into the myocyte. ${ }^{3)}$ Since $\beta$-oxidation of FFA is inhibited by the limited supply of oxygen to the ischemic heart, fatty acid derivatives, such as long chain acyl carnitine and long chain acyl CoA, increase in the myocyte. ${ }^{2)}$ Therefore, elevated FFA leads to the accumulation of long chain acyl carnitine and long chain acyl CoA in the presence of myocardial ischemia. The accumulation has been suggested as a cause of cellular damagc and ventricular arrhythmias in the ischemic heart. ${ }^{31}$ The administration of exogenous palmitoyl carnitine, which is a long chain acyl carnitine, decreases maximum diastolic potential, amplitude, $V_{\max }$ and action potential duration in canine Purkinje fibers. ${ }^{16)}$ POCA, an inhibitor of carnitine acyltransferase, prevents the accumulation of long chain acyl carnitine in hypoxic myocytes and markedly attenuates the electrophysiologic derangements induced by hypoxia assessed by intracellular transmembrane action potential recordings. ${ }^{5}$ Carnitine is essential for the transport of fatty acyl derivatives to the site of $\beta$-oxidation in the mitochondria, and itself improves mechanical and electrophysiological function in the ischemic heart. ${ }^{17}$ These experimental results indicate that it may be beneficial to reduce long chain acyl carnitine and long chain acyl $\mathrm{CoA}$ in the ischemic myocardium. In this study, procainamide and disopyramide prevented the accumulation of long chain acyl carnitine and long chain acyl CoA in the ischemic myocardium. Pande et al reported that quinidine, disopyramide and lidocaine prevented the increase in plasma FFA concentrations in the cat. ${ }^{9)}$ Siddle et al reported that local anesthetics, such as lignocaine and dibucaine, inhibited hormonestimulated lipolysis in adipose tissue." Mirro reported that quinidine, procainamide and disopyramide reduced the cyclic AMP content of guinea pig atria. ${ }^{6}$ Quinidine, procainamide and disopyramide produced concentrationdependent reductions in atrial rate which were paralleled by reductions in atrial cyclic AMP concentration. A number of investigators have proposed that myocardial infarction induces the release of catecholamines from both adrenal glands and sympathetic nerve terminals, and hence stimulates both 
peripheral and intramyocardial lipolysis which is mediated by cyclic AMP. The precise mechanisms by which procainamide and disopyramide prevent the accumulation of fatty acyl derivatives in the ischemic myocardium are unknown. It was suggested that the mechanisms which prevented the accumulation of fatty acyl derivatives might be the inhibition of lipolysis and the reduction of uptake into the myocyte as a result of decreased circulating FFA concentration. The inhibition of lipolysis may be influenced, in part, through alterations in the adenylate cyclase-cyclic AMP system. The results showed that procainamide and disopyramide have beneficial effects on FFA metabolism and it was suggested that one of the antiarrhythmic mechanisms of these drugs might be the prevention of the accumulation of FFA derivatives in the ischemic myocardium. The present study points out that knowledge of the biochemical effects of antiarrhythmic drugs may provide further insight into the mechanisms of action of these drugs.

\section{REFERENCES}

1. Oliver MF, Kurien VA, Greenwood TW: Relation between serum-free-fatty-acids and arrhythmias and death after acute myocardial infarction. Lancet I: 710, 1968

2. Liedtke AJ, Nellis S, Neely JR: Effects of excess free fatty acids on mechanical and metabolic function in normal and ischemic myocardium in swine. Circ Res 43:652, 1978

3. Russell DC, Oliver MF: Free fatty acids, catecholamine and arrhythmias in man. in Myocardial Ischemia and Lipid Metabolism, ed by Ferrari R, Katz AM, Shug A, Visioli O, Plenum Press, New York, London, p 307, 1984

4. Knabb MT, Saffitz JE, Corr PB, Sobel BE: The dependence of electrophysiological derangements on accumulation of endogenous long-chain acyl carnitine in hypoxic neonatal rat myocytes. Circ Res 58: 230, 1986

5. Opie LH: Propranolol and experimental myocardial infarction: substrate effect. Postgrad Med J 52 (suppl IV): 124, 1976

6. Mirro MJ: Effects of quinidine, procainamide and disopyramide on automaticity and cyclic AMP content of guinea-pig atria. J Mol Cell Cardiol 13: 641, 1981

7. Siddle $K$, Hales $N$ : The action of local anaesthetics on lipolysis and on adenosine $3^{\prime}: 5^{\prime}$ cyclic monophosphate content in isolated rat fat cells. Biochem J 142: 345, 1974

8. Illiano G, Chiosi E, Draetta GF, Laurenza A: Relationship between the fluidity of the membrane lipids and the activity of the membrane-bound proteins. The effects of lidocaine on the adenylate cyclase activity of rat myocardium. Gen Pharmac 14: 669, 1983

9. Pande M, Bhatnagar M, Pandey VG, Kar K, Dhawan BN: Effect of antiarrhythmic drugs on serum free fatty acid levels during experimental cardiac arrhythmias in the cat. Pharmacol Res Commun 15: 951, 1983

10. Prasad K, Weckworth P: Influence of glucagon on the cardiovascular effects of procainamide. Angiology 28: 515, 1977

11. Marquis NR, Fritz IB: Enzymological determination of free carnitine concentrations in rat tissues. J Lipid Res 5: 184, 1964

12. Veloso D, Veech RL: Stoichiometric hydrolysis of long chain acyl-CoA and measurement of the CoA formed with an enzymatic cycling method. Anal Biochem 62: 449, 1974

13. Kobayashi A, Nagao B, Nishiyama T, Yamashita T, Ishizaka K, Abe M, Hayashi H, Kamikawa $T$, Yamazaki N: Antiarrhythmic and myocardial metabolic effects of verapamil during 
coronary artery reperfusion. Jpn Circ J 49: 316, 1985

14. Lown B, Wolf $\mathrm{M}$ : Approaches to sudden death from coronary heart disease. Circulation 44: 130,1971

15. Carmelict E, Saikawa T: Shortening of the action potential and reduction of pacemaker activity by lidocaine, quinidine, and procainamide in sheep cardiac Purkinje fibers. An effect on $\mathrm{Na}^{+}$or $\mathrm{K}^{+}$currents? Circ Res 50: 257, 1982

16. Corr PB, Snyder DW, Cain ME, Crafford WA Jr, Gross RW, Sobel BE: Electrophysiological effects of amphiphiles on canine Purkinje fibers: implications for dysrhythmia secondary to ischemia. Circ Res 49: 354, 1981

17. Hayashi H, Suzuki Y, Abe M, Nishihara K, Masumura Y, Kamikawa T, Kobayashi A, Yamazaki N: Effects of L-carnitine on action potential of canine papillary muscle during hypoxic perfusion. J Electrocardiol 17: 85, 1984 